\title{
Wearable technology for baby monitoring: a review
}

\begin{abstract}
Sudden Infant Death Syndrome (SIDS) is one of the major reasons for infant death. Millennial parents' growing concern of SIDS has resulted in the springing up demand for health care services, and the invention of wearable baby monitoring systems. The development of wearable electronics and communication technologies has intensified the potential use of wearable technology in healthcare purposes. As infants are vulnerable to sleeping conditions, effective health monitoring using wearable technology can detect unexpected fall of respiratory rate, heart rate, and oxygen level and alleviate parents' anxiety by notifying sudden critical situations. The purpose of this review paper is to explore and summarize recent developments in the field of baby monitoring wearable technology and their functions to acquiring real-time vital signs. Technological breakthroughs in the field of physical biosensors and electronics integrate into textile materials and their applications in monitoring human vital signs such as heart rate, oxygen level, blood pressure, body temperature, and respiratory rate have discussed. A brief introduction to future challenges and recommended considerations during designing baby healthcare wearable technology has also covered.
\end{abstract}

Keywords: SIDS, baby monitoring system, health monitoring, healthcare, vital signs, wearable technology, smart textile
Volume 6 Issue 4 - 2020

\author{
Md Nakib UI Hasan, loan I Negulescu \\ Department of Textiles, Apparel Design, and Merchandising, \\ Louisiana State University, USA
}

Correspondence: Ioan I Negulescu Grace Drews Lehmann Professor, Department of Textiles, Apparel Design, and Merchandising, Louisiana State University and LSU AgCenter, Tel. (225) 578-1684, Email inegule@|su.edu

Received: June 16, 2020 | Published: July 14, 2020

\section{Introduction}

Sudden Infant Death Syndrome (SIDS) is the unexpected death of healthy babies who are less than a year old. SIDS is also known as crib death one of the most common cause of death in the United States ${ }^{1}$ with unexplained reasons which occur during the first year of an infant's life. In a study, Moon and Task Force on Sudden Infant Death Syndrome, ${ }^{2}$ reported that every year in the US nearly 3500 infants die from sleep-related reasons which include ill-defined deaths, SIDS, and accidental choking and suffocation on the bed. American Academy of Pediatrics has identified three main risk factors that are believed to be the cause of SIDS. ${ }^{3}$ Firstly, vulnerable babies having low birth weight, or who are premature, or whose mother smoked before their birth or with unknown genetic factors. Commonly, 2-4-month-old babies are at the highest risk as $90 \%$ of total SIDS is reported to occur before 6 months of age. Thus, age is the second factor. The third risk factor is infants sleeping position or bedding or other things that parents can change like parents smoking, and recent infections. Findings show that infants sleeping on their bellies have comparatively more risk of SIDS than infants who sleep on their backs. Parents have been advised to use separate bedding without blanket or pillows which can cover the baby's face during sleeping. However, modern millennial parents are highly worried and anxious about the risk of SIDS or any other disorder that might limit movement and breathing, looking for wearable technology to have assistance. In many countries increasing the successful use of wearable technology has been noticeable to monitor health vitals of elderly people to avoid sudden critical situations and ensure emergency medical support., ${ }^{4,5}$ Successful clinical trials have been reported on the use of wearable technology neurological, pulmonary, and cardiovascular patients. Like elderly persons, infants' continuous health monitoring can provide crucial biophysical parameters to allow urgent take care or adequate medical treatments. This measurement and monitoring can also facilitate physicians as well as scientists to understand and estimate many different pathological conditions and physical development of a child. ${ }^{6}$ Due to the recent development of wearable technologies, its application in entertainment, sports, healthcare, and military purposes is very common. Especially, for health monitoring, wearable technology can be subdivided into medical devices and consumer's products for home-usage. Among the consumer products fitness wearable devices are designed to track and monitor daily fitness conditions like steps, calories burned, diet, and sleep patterns. Among many popular fitness wearables Fitbit, Apple Watch, and Jawbone are usually for the young healthy amateur sportsman or athletes. The usage of both medical and commercial wearables are widespread for the neonatal, ${ }^{7}$ infant, ${ }^{8}$ athlete, ${ }^{9}$ or even elderly persons. ${ }^{10}$

Wearable devices have become increasingly popular throughout the world. The wearable technology market is predicted to hit a staggering $\$ 56.8$ billion by $2025,{ }^{11}$ where the market share of the global baby monitoring system will project to reach $\$ 1.63$ billion. $^{12}$ Baby monitoring devices are common in the form of onesies, socks, buttons, leg bands, and diaper clips. Sensors integrated with clothing or garments can measure vital biological signs such as pulse rate, respiration, and/or blood oxygen saturation that have recently been popular among parents. ${ }^{6,813}$ Parents can access their baby's health information statistics by using a smartphone or other digital devices. Despite increasing business, adoption, and continued to use the primary challenge of wearable technology is the extended future use. ${ }^{14}$ Most customers show their interest in these products because of their innovative, and attractive features. According to Karahanoğlu and Erbug,${ }_{15}^{15}$ perceived quality attributes can intensify the product adoption in the first impression, however, continued use is always determined by experience gathered during use..$^{14}$ Anderson and Lee ${ }^{16}$ suggest that researching factors influencing consumers' adoption and incorporating technical features accordingly can be the key to success for wearable manufacturers. Surprisingly, no known research has been conducted to review this baby monitoring wearable technology. This research is an attempt to review the basic working principle of wearable technology which is used to monitor baby health vitals. Focuses on the recent developments in the wearable sensors systems, and challenges needed to address for future development will also be given in this paper. 


\section{Literature review}

Wearable technology, wearables, or wearable devices is incorporation electronics into clothing or accessories that can be worn on a user's body. ${ }^{17}$ The purpose of wearable technologies is to provide entertainment, healthcare, and education in people's daily lives. Healthcare wearable, for example, Google Glass, Fitbit, Apple Watch, and Owlet Smart Sock have widely been using to collect health information. Wearable technology has been revolutionizing healthcare through wearable devices by monitoring health continuously outside of the medical or clinic. Previously healthcare monitoring was specific in hospitals and was involved with sophisticated electronic devices where many cables and sensors connected to the electronic display to observe patients. ${ }^{10}$ Resulting in patients' discomfort from restricted mobility. Moreover, these pieces of equipment needed qualified medical personnel to operate. Wearable technology, on the other hand, has integrated tiny electronic devices to cloth pieces most of which can work wirelessly and automatically send tracked bio-signals to medical staff. Therefore, healthcare practitioners have started using wearable devices for patient monitoring nowadays. The development of the algorithm for wearable is also facilitating the automatic prediction of many health-related prognostications, and interventions. ${ }^{18}$ Monitoring bio-signals is the medical process of collection, and analysis of respiratory, cardiovascular, and body temperature data which can be used to identify critical conditions and prevention of unexpected complications. ${ }^{10}$ The biological signal values usually over the range of normal range can be considered to be the cause of disease and this data can be a major source of information to evaluate patient's improvement of physical condition. The biomedical signals are measured using both invasive and noninvasive way. For this purpose, bio-sensors can be integrated into wearable materials including shirts, socks, bracelets, and buttons. Electronic circuits miniaturization allowing perfectly consistent and coherent incorporation of functionalities, hence, reducing bionic stigma and embrace the market potential of wearable. ${ }^{19}$

Devices with less complexity used to monitor health parameters do not require medial specialized medical qualification to use. Modern health monitoring devices can be used by experts or non-experts or even by the patients themselves in hospitals or at home. Noninvasive monitoring electronic devices sensors or actuators can be integrated with textile structures which can be in the form of clothing. Wearable electronic devices for baby monitoring are in the category of both devices and e-textiles. ${ }^{10}$ Commercially available baby monitoring wearable comes in several forms, with wireless electronics integrated into socks, leg bands, buttons, onesies, or diaper clips. These devices can send data to parents' smartphones using Bluetooth technology. ${ }^{20}$ Wearable structures track temperature, humidity, heart rate, and many more bio-signals. Hence, these structures needed to be smaller in size, easier to wear, and comfortable for the user. The baby monitoring wearable is a modular system based on different body areas for determining vital health signs. The whole monitoring process has three steps: skin the first sensor interface; sensor the second interface with garments and last interface is garment containing conductive yarn or conductive coating to establish the interconnection of electronics. The monitoring process consists of three main steps as illustrated in Figure 1; firstly, sensing and collecting data from physiological activities, secondly, relay data to cloud storage through the information communication system, and thirdly, analysis of data to extract clinically relevant data and representing it in a readable format. Advancement of sensor technology, microelectronics, information tech, and data analysis has facilitated the rapid wearable health monitoring devices development ${ }^{4}$ ranging from maternal, neonatal ${ }^{18}$ to elderly persons. ${ }^{21}$ Real-time data monitoring reduces parents' stress during sleeping when most parents are worried about SIDS or other sleep-related deaths or during working or are even when they far away or in the workplace. The audio-visual communication between parents and their children is a big sigh of relief, especially for anxious new parents.

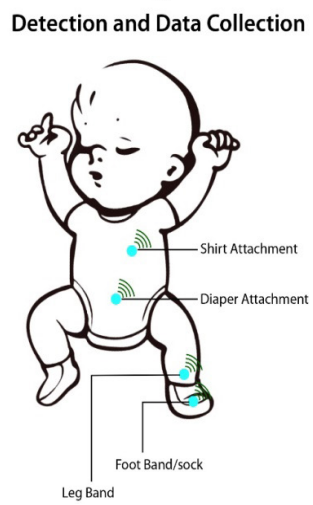

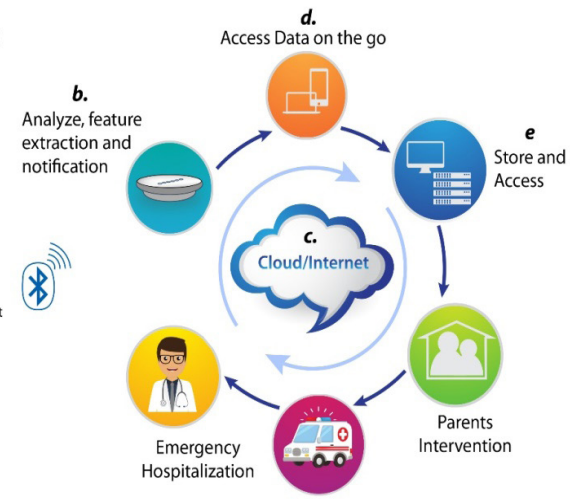

Figure I Illustration of working principle of baby health monitoring wearable technology a) Detection of vital health signs via body-worn wireless sensor integrated wearable; b) wirelessly connected base station collects healthrelated information and send back to both parents cellphone and internet cloud; c) transmitted data stored in the cloud which is accessible from anywhere; d) parents get notification directly from the base station as well as from cellphones in any unexpected fall down of baby vitals beyond the thresholds. Then emergency hospitalization if needed.

The literature of this review was identified using the following keywords: "infant care" "child care" "baby monitoring" "SIDS" "SIDS prevention" "use of technology to prevent SIDS" "healthcare wearable" "smart clothing" "wearable technology" "wearable tech for baby monitoring" etc. the purpose of this research is to provide an insight on the baby monitoring wearable technologies currently available to measure vital signs of 0 months to 18 -month babies. The resulting papers interconnected with citations in search results, our focus was primarily on the hardware and routinely monitored vital health signs and associated methods. Finally, we discuss some of the considerations and forthcoming future trends for the construction of wearable technologies for baby monitoring.

\section{Wearable technology for baby monitoring}

Baby monitoring technology includes different types of products such as movement monitor, audio-video monitor, health vitals monitor with or without an audio-video facility. The movement monitors are sensitive and can detect even the slightest movements of a baby and generate an alarm when a baby does not move. Mat monitor is a type of movement monitor where sensors are integrated under the mattress. This baby monitor generates an alarm if a baby stops movements for 15 to 20 seconds. Audio and video baby monitoring is another type of baby monitoring system to observe a baby without tracking baby vital signs. However, these babies monitoring have some constraints of realtime data monitoring, lack of fitting, and inaccuracy of information. To solve all these difficulties wearable baby monitoring technology has emerged. Currently, smartphones, smart watch, and smart clothing are the main wearable products available in the market with healthcare functions. ${ }^{22}$ Smartphones are ubiquitous with a big screen allowing 
effective human-computer interactions. Smart watches can ensure continuous contact with human skin providing health monitoring data continuously. Whereas smart clothing offers more precise and spontaneous health vitals monitoring. ${ }^{23,24}$ Both smart watch and smart clothing can be connected to the smartphone to review the whole health statistics which provides suggestions for improvement also. Therefore, most of the wearable baby monitoring technology is either in the form of wearable devices or smart clothing while both of these products can be wirelessly connected to smartphones. Bodily worn baby monitoring products to ensure better contact with the baby body and efficiently collect baby health vitals and transmit real-time data to connected devices. In the following section, we discuss, to date, the most popular health monitoring smart wearable systems, their functions, and operational workflow. A summary of the notable features of wearable baby monitoring products has been given in Table 1 at the end of the description.

\section{Levana oma sense}

Levana is a Canadian company founded by Raj Jain. The company has started producing baby monitors from 2001 and is the first color screen baby monitor producer in North America. Levana Oma Sense is a wearable device for baby breathing and movement monitor shown in Figure 2(a) with a dimension of 2.4 inches $(\mathrm{H})$ $X$ 1.92inches (W) X 1 inch (D). The device uses patented wakeUp technology to stimulate baby after sensing no movement for about 15 seconds. The randomized vibrations and alerts reestablish the baby's regular movement. Alarm set off if the regular movement does not return within 5 seconds followed by an emergency alarm and LED lights which signify parents' attention is needed for the baby. Levana Oma Sense is completely cordless and without the use of wireless frequencies. This $31.4 \mathrm{~g}$ (lightweight) device is portable and can easily be attached in the front of a baby's diaper (Figure 1), pants, or buttonup pajamas which ensure zero skin contact. Medical-grade ABS and TPE polymers use in Oma Sense provide excellent comfort needed for the baby's delicate skin. Levana has three upgraded versions of baby monitoring i.e. the Astra, the Alexa, and the Ayden. Many updated features including monitor, multi-colored LED light, and researchable battery with long-lasting (up to 48 hours) power. The wakeup technology stimulates a baby when it stops breathing, and ideally, the technology kick starts a baby's breathing pattern again. Levana Oma Sense sound output is 70 decibels at $10 \mathrm{~cm}$ and 60 decibels at $30 \mathrm{~cm}$ distance. Considering a general comparison with a disco generating 100 decibels at 1-meter distance. Potential drawbacks of this device are problems associated with attaching to clothing that doesn't have an opening over the belly, not having a smartphone app, and limited functionality during traveling.

\section{Snuza hero}

Snuza Hero is a wearable device designed to monitor a baby's abdominal movements. The device is shown in figure 2(b) made of silicone, thermoplastic elastomer (TPE), and medical grade polycarbonate integrated with a motion sensor. The device is of 1.7 inches (W), 1.1 inches (D), and 2.9 inches (L) size and can easily be attached to the baby diaper, as depicted in Figure 1. The ultra-sensitive flexible tip is designed to notice the slightest movements of the abdomen and sleep activity of a baby. Usually, it measures a threshold of more than 8 movements per minute and less than that can generate an alert. When a baby does not give any movement for 15 seconds, Snuza Hero gently vibrates to rouse the baby. Often the vibration is enough to rouse the infant and the Snuza Hero automatically revert to monitoring mode. Moreover, if its vibrations or the effort of rouse fail and the baby does not resume abdominal movements for an additional 5 seconds it generates an audible alarm to alert the parents warning that breathing has not been detected for 15 seconds. The Snuza Trio Plus Baby is an upgraded version of Snuza wearable technology for baby monitoring. This is a two-way audiovisual system that enables to monitor baby movement, with room temperature display. Parents can talk back to their baby through the built-in microphone and speakerphone and watch in a 3.5 -inch color display. ${ }^{25}$ Snuza is designed to pick up external movements and therefore, co-sleeping or bed-sharing is not advised during the use of Snuza. Additionally, when the baby is on a car seat or a stroller the motion does not allow Snuza to monitor the baby properly.

\section{MonBaby}

MonDevices is a wearable technology company based in New York City, USA widely known as a wearable MonBaby manufacturer. MonBaby baby monitor invented by Vaitaitis in 2014. Inside the MonBaby smart button presented in Figure 2(c) is a circuit board containing tiny surface mount electronic components integrated with a low energy Bluetooth technology microprocessor chip. The small wireless smart button can easily fasten onto a baby's clothing article disregarding the size of the baby. The smart button tracks a baby's breathing, movement level, sleeping position, and fall detection. ${ }^{26}$ It can also monitor ambient temperature around the baby's body and protects the baby from overheating or cold. The bio-signals then transmit to the Bluetooth connected smartphone, tabs, or other devices. MonBaby is designed to take various proactive approaches from standard baby monitors by sending real-time updates of a baby's breathing, movement, and sleep statistics five times per second. ${ }^{27}$ MonBaby app which suits both iPhone and Android devices gives the whole report to parents' smartphones. The app alert can be adjusted for many different parameters. For instance, parents can set an alert when their baby stops breathing, or when the baby rolls onto his or her stomach or restless sleep so that parents can respond quickly. In 2014, MonBaby was awarded in the category of wearable smart clothing in the innovation world cup, Munich, Germany. ${ }^{25}$ The device has a limited range of Bluetooth reliance, and the motion-sensing is not very much reliable during the usage with a certain type of sleepers. However, the MonBaby smart button is a tiny piece of sensor integrated device efficiently monitors baby vitals emitting 1000 times less radio frequency than a typical cellphone.

\section{Mimo}

Mimo is a Boston, USA, based wearable smart clothing manufacturer for baby monitoring. The Mimo system contains two main components namely Turtle and Lilypad. Turtle as shown in Figure 2(d) is the wireless sensor magnetically attached to the baby clothing known as kimonos and the Turtle is connected to the Lilypad equivalent to the base station of Owlet baby monitoring system. Both Turtle and Lilypad use Bluetooth technology to connect to the cloud and smartphone. Mimo uses ultra-low power Bluetooth technology which consumes low energy and its encrypted technology to secure baby data. The Turtle detects baby breathing, baby position, sleep patterns, and skin temperature and sends realtime data to the Lilypad. ${ }^{26}$ The information then transfers to the cloud and finally to the parents' smartphones or other smart devices ${ }^{28}$ as illustrated in figure 1. Parents can customize the alert of irregular breathing, weak-ups, and roll-overs depending on the preferability. The kimonos are made of $100 \%$ cotton or tencel fibers ${ }^{29}$ and have machine dry-washability. Mimo app is compatible with both iOS and Android operating systems thus, parents can easily track baby vitals 
and get notifications in their cellphones during any abnormal events. The Turtle monitor needed to be within five feet of the Lilypad for better connectivity and baby must wear clothing articles are the major drawbacks. Preventing SIDS is associated with getting real-time vital signs while the device bounces those signals off a server and then transmits back to the smartphone. Thus, Mimo does not provide realtime health data which has significant importance especially in terms of babies having medical issues.

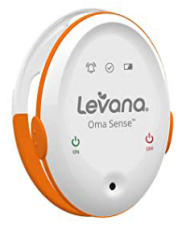

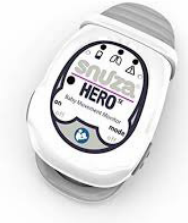

b

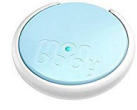

c

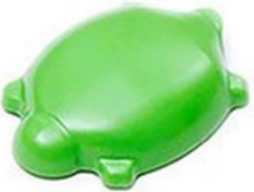

d

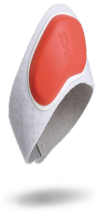

e

Figure 2 a) Levana Oma Sense in the form of diaper clip, b) Snuza Hero (SE) wearable baby abdominal movement monitor as diaper clip, c) MonBaby monitor as a shirt button and d) Mimo turtle as shirt attachment, e) Sproutling baby monitor.

\section{Owlet smart socks}

In 2013, Owlet Baby Care was founded to offer products to simplify parenthood. The Owlet baby monitor is a technology comprises of a soft Smart Sock and a base station. Pulse oximetry sensor, hospitalgrade noninvasive technology, embedded on the Smart Sock that fits snugly on baby's foot to monitor their heart rate and oxygen levels during sleeping. ${ }^{30}$ Bluetooth technology is used to connect the Smart Sock to the base station and the base station to the smartphones or other devices. The sensor attached to a specially designed Smart Sock collects and sends vital health signs to the base station. Then, the information directly communicates to connected smartphones and the internet cloud. The base station glows green which represents everything is okay i.e. heart rate (60-220 BPM) and oxygen levels $(\geq 95)$ are within the normal range. However, in any event, when a baby's heart rate or oxygen levels falls too low or high, the alarm on the base station will go off with flashing red light and app notification to parents' cellphones (Figure 3). Owlet baby monitor helps parents anxiety-free sound sleep who is worried about the frightening possibility of sudden infant death. ${ }^{13}$ The convenient design feature of the sock is easily adjustable and can fit easily to infants from $0-18$ months. Socks are manufactured by interlocking, a process of weft knitting, ${ }^{31}$ comb yarns which are made of finer, smoother, cleaner, soft, and strong fibers. The high hairiness of the combed yarn ${ }^{32,33}$ assures comfort to the baby's foot. Furthermore, cotton fibers' additional property of sweat absorbency, ${ }^{34}$ breathability, and heat-retention provide the desired comfort ${ }^{35}$ to the baby during prolonged use. In 2016, Owlet sold 150,000 units of smart socks, which generated $\$ 19$ million in revenue. ${ }^{13}$ A recent study on 47,495 Owlet users has reported $96 \%$ of the parents felt less anxious and $94 \%$ expressed better sleep quality during the use of Owlet Smart Sock. ${ }^{8}$ However, a large number of parents have also reported on parent enhanced anxiety due to frequent false alarm, app crashing, data security, and real-time data transmission.

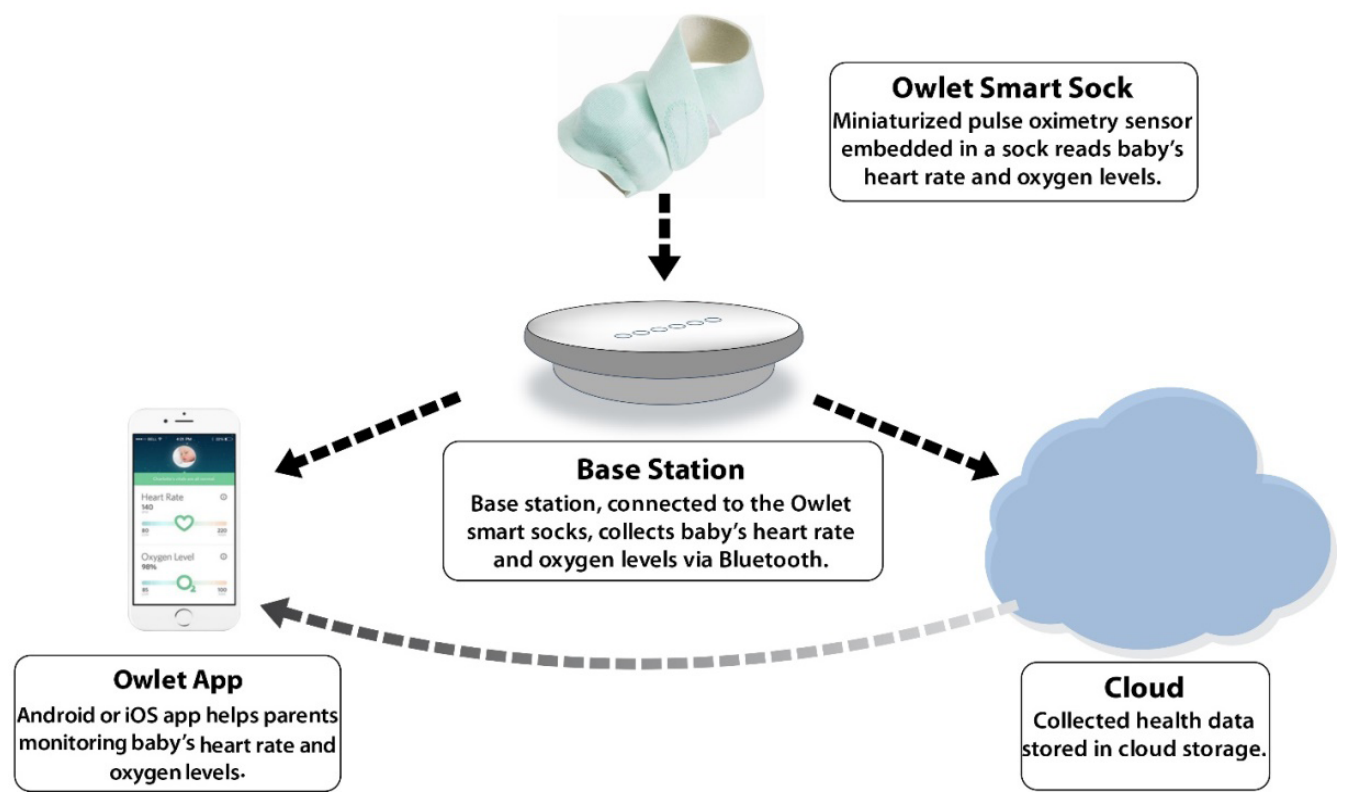

Figure 3 Owlet smart sock and its workflow. 


\section{Nanit plus}

Nanit Plus is a smart baby monitoring technology that uses computer vision and machine learning to track baby health vitals. The main components include a Nanit swaddle and an overhear video camera. The Nanit swaddle is made of $100 \%$ cotton which can easily be wearable over any other baby shirts of pajamas. It contains custom-designed patterns of images (Figure 4) which is observed by the overhead video camera to detect baby movement and sleep pattern. Collected real-time sound, and motion data transferred to the parents' smartphone or other smart devices through Bluetooth connection. Even when the internet is down the Nanit continues to stream over Wi-FI sending a perfectly framed view of the baby crib. ${ }^{36}$ The Nanit Plus camera is integrated with a two-way audio system which allows parents to speak or sing to their baby. Nanit plus is Alexa enabled device that facilitates parents to see the baby's live stream or ask to find out the sleep pattern without intervention. As the Nanit swaddle does not contain any electrical components integrated into it, therefore, is not considered as a wearable technology rather a camerabased baby monitoring system.

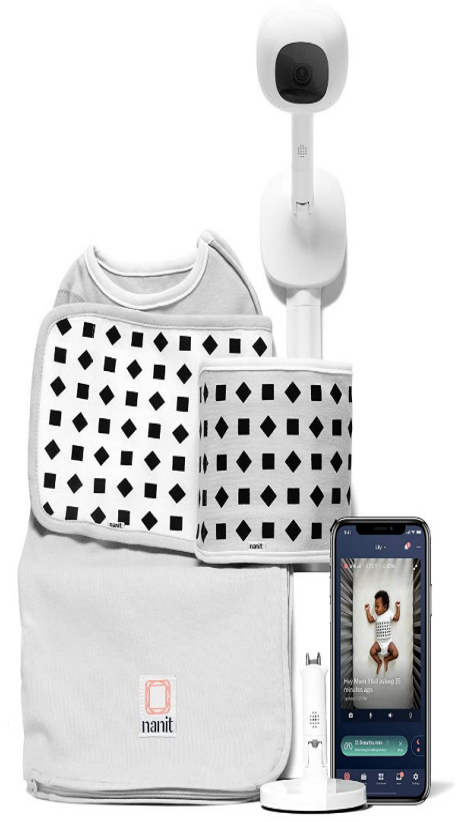

Figure 4 Nanit plus baby monitoring system.

\section{Sproutling}

Sproutling is a wearable device in the form of a band that can easily be wrapped around a baby's ankle. The most important components are the wearable sensor, smart charger, and band. The wearable sensors are integrated into a soft and gentle strap made of soft, red silicon. Utilizing machine learning the device as depicted in Figure 2(e) can determine a baby's body movement and sleep patterns. ${ }^{37}$ The device is paired with a base station known as the charging bowl and the charging bowl to the smartphones. The sensor detects, measures, and sent real-time data to parents' smartphones via the charging bowl similar to the illustration in Figure 1. The base station can also analyze the ambient temperature and the level of noise of the room. ${ }^{38}$ Both iOS and android enable sprouting app to give notifications about baby sleep, wake up, and time to take care of. The synthesized data then converted into an easy-to-understand statistical format in the smartphone or other devices. This wearable is completely wireless and can easily be charged on its charging pad which ensures long-lasting and continuous use. Each Sproutling has a unique digitally signed token to permit authorized users and security of information. Research findings show that Sproutling baby monitor generated frequent false alarm while the baby had normal health vitals. ${ }^{39}$ Currently, the Sproutling baby monitoring system is not available in the market.

\section{Tracking bio-physical information}

In common practice medical professionals measure four major health information, heart rate, respiration rate, blood pressure, and body temperature which give a sporadic snap of patients' health conditions. Baby monitoring wearables are subjected to measure a baby's vital health signs (Table 3 ) to reduce the potential risk of sudden infant death. In the following section, we summarize the activities of the baby monitoring technology and the recent development of textile-based sensors and electronics to accomplish more accurate and precise functionalities. Commonly used wearable sensor types and their functions have been presented in Table 2.

\section{Body temperature}

Commonly used body temperature measuring is done by the thermoelectric effect, thermistors, or by optical means. ${ }^{40}$ Among these methods, thermistors are primarily used in wearable because it is non-invasive. The thermistors having the quality to be embedded into textiles should be highly static and dynamic in accurately detecting human skin temperature in the range of $30^{\circ} \mathrm{C}-45^{\circ} \mathrm{C}$. Conductive textile wires have been used with a negative temperature coefficient resistor which has finally been integrated with a wearable smart jacket to monitor neonatal. ${ }^{41}$ Nickel and tungsten wires were successfully been knitted into fabric structure to measure the skin temperature of wearers. ${ }^{42}$ Poly (3, 4-ethylene dioxythiophene) poly (styrene sulfonate) (PEDOT: PSS), silver nanoparticles (AgNPs), and graphene ink composite can be fabricated to produce self-powered temperature sensor with high stretchability. Printing on stretchable knitted fabric with thermoelectric materials is the least expensive process to produce temperature sensors. ${ }^{43}$ Temperature sensing yarn containing thermistor and interconnected copper wire encapsulated in resin wrapped by fibers with knitted sheath has successfully been applied to manufacture sock to measure feet temperature particularly of diabetic patients. ${ }^{44}$ Many thermistors and temperature integrated circuits are now available in the market. LM35, for instance, is a widely used thermistor.

\section{Heart rate}

Heat rate measurement can be done by electrical or optical to strain sensors. The electrocardiogram (ECG) which also known as myocardium is a measurement of the electrical activity of the heart muscle through a skin surface measurement. Corresponding to the change of heartbeat icons move through the heart muscle and a change of the gradients is made. Differential measurement of the electrical potential on the body surface at different locations of the body generates different ECG leads. The ECG leads are usually formed from three dissimilar electrodes placed on the body, with an optional fourth electrode used as a reference. ${ }^{45}$ Many researchers are trying to design and develop textile electrodes to integrate into clothing and 
provide an ECG. In a collaborative effort, Catrysse and Bekintex have developed knitted and woven stainless electrodes which are known as Textrodes, an alternative to conventional ECG electrodes. ${ }^{46}$ Textrodes are less irritating compared to gel electrodes and the less toxic stainless steel yarn can be manipulated as textile material with washability. However, Textrodes give poor skin-electrode contact and increased sensitivity to patient-based motion artifacts. ${ }^{47}$ In research to prevent sudden infant's death, prototype Textrodes integrated to a flexible printed circuit and an embroidered on an elastic belt and a baby suit, respectively, with wireless powering and data communication, were developed ${ }^{47}$ This alternative ECG of the new electrode exhibit a lower signal-to-weight ratio compared to traditional gel electrodes with beat-to-beat heart rate detection. To endure better contact between human skin and electrodes application of embroidery technology on the textile surface resulted to have better results. ${ }^{48}$
To measure the heart rate, wearable devices like Fitbit, Owlet smart socks, and Apple Watch use plethysmography, an optical modality. ${ }^{49} \mathrm{~A}$ light-emitting diode (LED), placed close contact with the skin, shines a light directly on the arterial blood vessel. Photodiodes detect the reflected light. Minimum intensities of reflected light are observed during the contraction of the chambers of the heart to drive blood into the aorta and pulmonary artery while the highest light absorbed by the oxygenated hemoglobin. The heart rate and blood oxygen saturation $\left(\mathrm{SpO}_{2}\right)$ measurement is primarily used in the critical care unit while in case wearable devices continuous monitoring can vary depending on motion, skin contact, and power consumption. Study shows that organic light-emitting diodes (OLEDs) and an organic photodiode (OPD) have been used to develop flexible pulse oximeter which exhibits similar heart rate estimation compared to a commercially obtainable pulse oximeter. ${ }^{50}$

Table I Summary table of baby monitoring wearable technology products and features

\begin{tabular}{|c|c|c|c|c|c|c|c|}
\hline & $\begin{array}{l}\text { Levana } \\
\text { Oma Sense }\end{array}$ & Snuza Hero & MonBaby & Mimo & $\begin{array}{l}\text { Owlet } \\
\text { Smart Sock }\end{array}$ & Nanit Plus & Sproutling \\
\hline Form of the wearable & Diaper clip & Diaper clip & Button & Shirt attachment & Sock & Nanit swaddle & Leg band \\
\hline Pulse rate & No & No & No & No & Yes & No & Yes \\
\hline Respiratory motion & Yes & Yes & Yes & Yes & No & No & No \\
\hline Pulse oximetry & No & No & No & No & Yes & No & No \\
\hline Skin temperature & No & No & No & Yes & No & No & Yes \\
\hline Ambient temperature & No & No & Yes & No & No & No & Yes \\
\hline Body movement & Yes & Yes & Yes & Yes & No & Yes & Yes \\
\hline Body Position & No & No & No & Yes & No & No & Yes \\
\hline Sleep pattern & No & No & Yes & Yes & No & Yes & Yes \\
\hline Machine dry-washability & No & No & No & Yes & Yes & Yes & $\mathrm{N} / \mathrm{A}$ \\
\hline Cloud technology & No & No & Yes & Yes & Yes & Yes & Yes \\
\hline Uses App & No & No & Yes & Yes & Yes & Yes & Yes \\
\hline $\begin{array}{l}\text { Bluetooth/Wi-Fi } \\
\text { connectivity }\end{array}$ & No & No & Yes & Yes & Yes & Yes & Yes \\
\hline Cost, $\$$ & 125 & 80 & 170 & 350 & 250 & 294 & 300 \\
\hline
\end{tabular}

Table 2 General form of wearable sensors and their functions

\section{Biochemical signal biosensors \\ - $\quad$ Glucose monitoring \\ - Sweat, tear, saliva analysis}

Temperature sensor

- Body temperature detection

\author{
Physical signal biosensor \\ - Micro-force detection \\ - Motion detection \\ - $\quad$ Pulse weave measurement \\ - Voice detection \\ - Brainwave detection
}

Multifunctional sensors

- Ambient temperature detection 
Table 3 Summarize of most common health vital signs

\begin{tabular}{ll}
\hline Parameter & Type of technology \\
\hline Body temperature & Thermistors \\
& Optical means \\
& Thermoelectric effects \\
Blood pressure & Capacitive strain sensors \\
& Piezoelectric capacitors \\
Rate of the heart & Skin electrode \\
& Optical \\
Respiration rate & Ml sensor \\
Pulse oxygenation & Optical means \\
\hline
\end{tabular}

\section{Respiration rate}

Direct airflow detection during breathing or indirect measurement of expansion and contraction of the chest and abdomen can be used to determine and monitor the respiration rate. Textile sensors are inconvenient indirect measurements of breath flow because of its placement near to the nose or mouth. ${ }^{51}$ Due to the rapid development of textile-based sensors, conductive knitted fabric based on a loop structure under biaxial extensions have been introduced to measure indirect lung movement during inhalation and exhalation. ${ }^{21}$ For longterm respiration rate monitoring a coated piezoresistive sensors have been integrated into apparel as a part of wearable sensing. ${ }^{52}$ Another research claim to develop a respiration belt using a textile-based strain sensor which is a combination of silver-coated polymeric yarn with elastomeric yarn and together knitted into the fabric..$^{53}$ Respiration rate monitoring provides important information on diseases like chronic obstructive pulmonary disease, asthma, sleep appease, and anemia. Sleep quality monitoring used to measure health quality and also a syndrome of sudden death and cardiopathies or heart diseases. ${ }^{54}$

\section{Blood pressure}

Sphygmomanometers are commonly used devices to measure blood pressure. The shortcomings of these devices for continuous monitoring lead to the development of capacitive sensitive strain sensors. ${ }^{55}$ This au courant blood pressure monitoring system uses compressible and piezoelectric capacitive strain sensors. The piezoelectric capacitive strain sensors are made of robust two flexible electrodes (in a sandwiched form) whereas the compressible capacitive strain sensors are made of an elastic. Externally applied pressure squeezed the dielectric which leads to the change of capacitance. Strain in the piezoelectric material induces voltage generation in the device. Blood pressure measurement can be performed attaching this device to the wrist, arm, or neck for continuous monitoring. ${ }^{21}$

\section{Pulse oxygenation}

Unspecific balance of blood oxygen level can lead to hypoxemia which is a major cause of human death over the world. Commonly, $95 \%$ to $100 \%$ blood oxygen level is viewed as normal while less than $90 \%$ is can cause hypoxemia. ${ }^{21}$ Tissue oxygenation $\left(\mathrm{StO}_{2}\right)$, peripheral oxygenation $\left(\mathrm{SpO}_{2}\right)$ and venous oxygenation $\left(\mathrm{SvO}_{2}\right)$ are mostly used blood oxygen level measuring method which varies both on the measurement location and procedure. $\mathrm{SpO}_{2}$ is a non-invasive method and is thus ubiquitous in wearable technology as well. A pulse oximeter is an optical approach containing two LED one of which shines a light (660nm wavelength) through a translucent part like a fingertip, earlobe, wrist, or area on the forehead and another is an infrared (940nm wavelength). Volume and concentration of oxy-hemoglobin in the blood manipulate light absorption. A photodiode located on the other side of the translucent part collects the transmitted light. Finally, a pulse oxygenation measurement $\left(\mathrm{SpO}_{2}\right)$ can be calculated based on Beer-Lambert's law. ${ }^{56}$ Advanced organic optoelectronics, flexible organic LEDs, and organic photodetector $\mathrm{OPDs}^{50}$ are have overcome constraints measurement location (only fingertip or ear lobes) of inorganic optoelectronics. Oxygenation measurement of all organic sensors can easily be mounted on a forearm using a flexible bandage or clothing that comprises the photodiodes and an OLED light source. ${ }^{57}$

\section{Challenges and future directions}

There are many challenges of wearable baby monitoring technology and smart textile-based sensors and electronics. The first and foremost challenge is the accuracy and consistency of health information measurement. Most of the wearable is not substantiated by scientific and peer-reviewed research and none of the companies have FDA approval. Hence, manufacturers should demonstrate evidence of safety and efficacy in healthcare through scientific peer-reviewed research. A false negative result from these devices could be a cause of potentially critical condition leading fatality while false positives can often lead to overtreatment and cause more anxiety than peace of mind. ${ }^{39}$ Realtime data transfer and synchronization is another important aspect of wearable technology. ${ }^{18}$ More accurate and uninterrupted data transfer without any delay can result in the best detection of critical events alleviating potential harmful interventions and patients' confusion. The wearable baby monitors should be compatible across all platforms like iOS and Android. Another important challenge of these wearables is the proper encryption and authentication mechanism to secure privacy and protect personal health information. ${ }^{58,59}$ Besides this, the uninterrupted power supply has remained a big issue as the sensors and data processing circuits consume high power. ${ }^{60}$ Additionally, current price of wearable baby monitoring is significantly high. So, enhanced accessibility with affordable price points might be helpful for parents with financial constraints. Significant improvement in the sensitivity, durability, reproducibility, and seamless integration of textile-based technologies is needed to ensure prolonged monitoring. In this multidisciplinary research, expert knowledge and skills from the field of material science, chemistry, manufacturing, information technology, and healthcare can efficiently address these research challenges. Besides this, data acquisition and artifacts removal, and data mining form the sensor are important as wearable sensors have limited sensitivity and reliability. Thus, filtering unwanted signals and precise measurement of health vital signs appeal to the need for expert knowledge from the data science discipline. Researchers, developers, and manufacturers must resolve these challenging issues which will improve the utility and expansion of the wearable technologies for long-term sustainable development. ${ }^{56-59}$

\section{Conclusion}

This paper has presented an overview of some recently developed commercial available baby monitoring wearable technology products and textile-based electronics for healthcare purposes. The development 
of computer science, wireless communication, biomedical sensing, and flexible wearable sensors has created an opportunity to develop constant health monitoring devices for neonatal to elderly people. ${ }^{60}$ Electronic sensor integrated wearable are primarily in the form of socks, bands, and bracelets that use a wireless communication system to connect parents. Integration with the smartphone has been supporting the growth of the industry as they enable the parents to monitor babies conveniently, and spontaneously. The increasing number of nuclear families and working parents in the USA, Canada, and other European countries have also been a reason fueling the adoption of the product. Furthermore, the rising purchasing power in developing countries like China, India, and Brazil is expected to spend more on children's safety. Tech-savvy millennial parents are embracing wearable smart devices as a means to prevent unexpected infant death or abnormal events. ${ }^{6}$ Studies show that many parents highly convinced about the functionalities of existing baby monitoring systems while some parents have reported more anxiety than relief. These smartphone-integrated baby monitoring devices are in the preliminary phase and have not yet received approval from the product regulatory organizations like the US Food and Drug Administration (FDA). Some wearable baby monitor manufacturers are putting their effort to get FDA approval as they envision selling their products as medical devices. The noninvasive wearable devices are expected to contribute to healthcare both personal and medical settings. Successful detection of heart rate, oxygen level, blood pressure, body temperature, and respiratory rate can reduce parents' dreadful continuous anxiety about their babies. The collection of health data and its management using wearable technology have the potential to reduce unexpected SIDS and make a perfect clinical decision, therefore, reduce frequent unnecessary visits to the hospital. Besides providing promised services needed for baby health monitoring the wearable technologies with consistent functionalities will eventually lead to sustainable healthcare and social benefit.

\section{Acknowledgments}

None.

\section{Funding}

None.

\section{Conflicts of interest}

The authors have no conflicts of interest regarding the publication of this paper.

\section{References}

1. Moreno MA. Reducing the risk of sudden infant death syndrome. JAMA Pediatrics. 2017;171(2):204.

2. Moon RY, Task Force on Sudden Infant Death Syndrome. SIDS and other sleep-related infant deaths: evidence base for 2016 updated recommendations for a safe infant sleeping environment. Pediatrics. 2016;138(5):e20162940

3. American Academy of Pediatrics Task Force on Sudden Infant Death Syndrome. The changing concept of sudden infant death syndrome: diagnostic coding shifts, controversies regarding the sleeping environment, and new variables to consider in reducing risk. Pediatrics. 2005;116(5):1245-1255.

4. Patel S, Park H, Bonato P, et al. A review of wearable sensors and systems with application in rehabilitation. Journal of neuroengineering rehabilitation. 2012;9(1), 21.
5. Golant SM. A theoretical model to explain the smart technology adoption behaviors of elder consumers (Elderadopt). Journal of aging studies. 2017;42:56-73.

6. Bonafide CP, Jamison DT, Foglia EE. The emerging market of smartphoneintegrated infant physiologic monitors. JAMA. 2017;317(4):353-354.

7. Bouwstra S, Chen W, Feijs L, et al. Smart jacket design for neonatal monitoring with wearable sensors. Sixth International Workshop on Wearable and Implantable Body Sensor Networks; 2009.

8. Dangerfield MI, Ward K, Davidson L, et al. Initial experience and usage patterns with the owlet smart sock monitor in 47,495 newborns. Global Pediatric Health. 2017;4:1-8.

9. Zhang M, Luo M, Nie R, et al. Technical attributes, health attribute, consumer attributes and their roles in adoption intention of healthcare wearable technology. International Journal of Medical Informatics. 2017;108:97-109.

10. Aileni RM, Valderrama AC, Strungaru R. Wearable electronics for elderly health monitoring and active living. In Ambient Assisted Living and Enhanced Living Environments: Elsevier; 2017:247-269.

11. Wearable Technology Market worth $\$ 56.8$ billion by 2025 with a growing CAGR of $11.28 \%$. January 2017.

12. Baby monitors market size to reach USD 1.63 Billion by 2025. 2017.

13. Sportelli N. Owlet's smart sock makes millions selling parents peace of mind -- but doctors are unconvinced. Forbes; 2017.

14. Shih PC, Han K, Poole ES, et al. Use and adoption challenges of wearable activity trackers. iConference, iSchools; 2015.

15. Karahanoğlu A, Erbuğ Ç. Perceived qualities of smart wearables: determinants of user acceptance. Proceedings of the 2011 Conference on Designing Pleasurable Products and Interfaces; 2011.

16. Anderson G, Lee G. Why consumers (don't) adopt smart wearable electronics. IEEE Pervasive Computing. 2008;7(3):10-12.

17. Wright R, Keith L. Wearable technology: If the tech fits, wear it. Journal of Electronic Resources in Medical Libraries. 2014;11(4):204-216.

18. Dunn J, Runge R, Snyder M. Wearables and the medical revolution. Personalized medicine. 2018;15(5):429-448.

19. Gonçalves C, Ferreira da Silva A, Gomes J, et al. Wearable e-textile technologies: A review on sensors, actuators and control elements. Inventions. 2018;3(1):14.

20. Callahan A. New type of baby monitors offers 'peace of mind' but may deliver just the opposite. The Washington Post; 2017.

21. Wang Z, Yang Z, Dong T. A review of wearable technologies for elderly care that can accurately track indoor position, recognize physical activities and monitor vital signs in real time. Sensors. 2017;17(2):1-36.

22. Tehrani K, Michael A. Wearable technology and wearable devices: everything you need to know. Wearable Devices Magazine; 2014.

23. Cherenack K, van Pieterson L. Smart textiles: challenges and opportunities. Journal of Applied Physics. 2012;112(9):091301.

24. Cheng J, Lukowicz P, Henze N, et al. Smart textiles: from niche to mainstream. IEEE Pervasive Computing. 2013;12(3):81-84.

25. Moore CJ. 8 best baby breathing monitors available on the market. October 10, 2019.

26. Wood M. Baby monitors for a smart nursery, but parents are still better. The New York Times; 2014.

27. O'Brien C. Tech tools review: MonBaby smart button. The Irish Times; 2016. 
28. Lee BY. Does your baby really need that monitor? Forbes; 2017.

29. Hasan NB, Begum AR, Islam A, et al. Tencel process optimization in conventional cotton processing machineries and a quality comparison with similar cotton yarn count. J Textile Sci Eng. 2017;7(3):1-6.

30. Harris H. Owlet baby monitor 2 review: smart sock or little crock? 2019.

31. Islam SMZ, Nakib-Ul-Hasan M, Rahman MM, et al. Comparative study of single jersey, rib and interlock fabric keeping constant yarn count and stitch length. GUB Journal of Science and Engineering. 2016;3(1):4754.

32. Shahid MA, Hossain MD, Nakib-Ul-Hasan M, et al. Comparative study of ring and compact yarn-based knitted fabric. Procedia Engineering. 2014;90:154-159.

33. Ul-Hasan N, Afroz F, Islam MM, et al. Comparative study of mechanical properties, tpi, hairiness and evenness of conventional ring and modern rotor spun yarn. European Scientific Journal. 2014;10(33).

34. Cruz J, Leitão A, Silveira D, et al. Study of moisture absorption characteristics of cotton terry towel fabrics. Procedia Engineering. 2017;200:389-398.

35. Hosseini Ravandi SA, Valizadeh M. Properties of fibers and fabrics that contribute to human comfort. In: Song G, editor. Improving Comfort in Clothing. Woodhead Publishing; 2011:61-78.

36. Fatherly. The nanit plus baby monitor is the next best thing to a night nurse. 2019.

37. Stolyar B. The Sproutling wearable baby monitor is officially on the market. 2017.

38. Seifert D. Sproutling's baby monitor of the future aims to put parents at ease. The Verge; 2014.

39. Bonafide CP, Localio AR, Ferro DF, et al. Accuracy of pulse oximetrybased home baby monitors. JAMA. 2018;320(7):717-719.

40. Khan Y, Ostfeld AE, Lochner CM, et al. Monitoring of vital signs with flexible and wearable medical devices. Advanced Materials. 2016;28(22):4373-4395.

41. Chen W, Dols S, Oetomo SB, et al. Monitoring body temperature of newborn infants at neonatal intensive care units using wearable sensors. Corfu, Greece: Proceedings of the Fifth International Conference on Body Area Networks; 2010.

42. Husain M, Kennon R. Preliminary investigations into the development of textile based temperature sensor for healthcare applications. Fibers. 2013;1(1):2-10

43. Hatamie A, Angizi S, Kumar S, et al. Textile based chemical and physical sensors for healthcare monitoring. Journal of Electrochemical Society. 2020;167(3):037546.

44. Hughes-Riley T, Lugoda P, Dias T, et al. A Study of thermistor performance within a textile structure. Sensors. 2017;17(8):1804.
45. MIT. Electrocardiogram measurement circuit design lab. 2013.

46. Catrysse M, Puers R, Hertleer C, et al. Towards the integration of textile sensors in a wireless monitoring suit. Sensors and Actuators A: Physical. 2004;114(2-3):302-311.

47. Coosemans J, Hermans B, Puers R. Integrating wireless ECG monitoring in textiles. Sensors and Actuators A: Physical. 2006;130:48-53.

48. Marozas V, Petrenas A, Daukantas S, et al. A comparison of conductive textile-based and silver/silver chloride gel electrodes in exercise electrocardiogram recordings. Journal of electrocardiology. 2011;44(2):189-194

49. Servati A, Zou L, Wang, ZJ, et al. Novel flexible wearable sensor materials and signal processing for vital sign and human activity monitoring. Sensors. 2017;17(7):1622.

50. Lochner CM, Khan Y, Pierre A, et al. All-organic optoelectronic sensor for pulse oximetry. Nature Communications. 2014;5(1):5745.

51. Folke M, Cernerud L, Ekström M, et al. Critical review of non-invasive respiratory monitoring in medical care. Medical and Biological Engineering and Computing. 2003;41(4):377-383.

52. Guo L, Berglin L, Wiklund U, et al. Design of a garment-based sensing system for breathing monitoring. Textile Research Journal. 2013;83(5):499-509.

53. Atalay O, Kennon WR, Demirok E. Weft-knitted strain sensor for monitoring respiratory rate and its electro-mechanical modeling. IEEE Sensors Journal. 2015;15(1):110-122.

54. Lokavee S, Puntheeranurak T, Kerdcharoen T, et al. Sensor pillow and bed sheet system: Unconstrained monitoring of respiration rate and posture movements during sleep. IEEE International Conference on Systems, Man, and Cybernetics; 2012.

55. Schwartz G, Tee BCK, Mei J, et al. Flexible polymer transistors with high pressure sensitivity for application in electronic skin and health monitoring. Nature Communications. 2013;4(1):1859.

56. Zhou M. Electronic technology in home medical equipment in Chinese market. (Bachelor of Industrial Management). Savonia University of Applied Sciences; 2016.

57. Bansal AK, Hou S, Kulyk O, et al. Wearable organic optoelectronic sensors for medicine. Adv Mater. 2015;27(46):7638-7644.

58. Gao Y, Li H, Luo Y. An empirical study of wearable technology acceptance in healthcare. Industrial Management \&amp; Data Systems. 2015;115(9):1704-1723.

59. Hasan MNU, Liu C. Mining reviews: Exploring consumer responses to smart clothing for healthcare. New Orleans, Louisiana: American Collegiate Retailing Association Conference; 2020.

60. Zhu Z, Liu T, Li G, et al. Wearable sensor systems for infants. Sensors. $2015 ; 15(2): 3721-3749$. 